\title{
43 Fettverteilungsmuster, ihre Messgrößen und das Demenzrisiko
}

\author{
(c) Springer-Verlag GmbH Deutschland, ein Teil von Springer Nature 2018 \\ D. Mathias, Fit und gesund von 1 bis Hundert \\ https://doi.org/10.1007/978-3-662-56307-6_43
}

Standardgröße für die Gewichtsbestimmung ist der Body-Mass-Index. Er errechnet sich aus der Körpermasse (kg) dividiert durch die Körpergröße $\left(\mathrm{m}^{2}\right)$; - Tab. 43.1.

Ein besseres Maß ist der "surface-based body shape index" (SBSI). Die Ermittlung dieses Oberflächen-Körperform-Index erfolgt über die vier Faktoren Oberfläche, vertikaler Rumpfumfang, Größe und Bauchumfang. Er berücksichtigt, dass auch eine starke Muskulatur gut trainierter Sportler/innen zu Übergewicht führen kann (Rahmann und Adjeroh 2015). Mit dem SBSI lassen sich die mit dem Älterwerden steigenden Gesundheitsrisiken besser abschätzen ( $\triangleright$ Kap. 47 ).

Die sich vermehrt bildenden Fettpolster werden aber nicht nur durch den BMI oder den SBSI charakterisiert. Von großer Bedeutung ist auch die Verteilung des überschüssigen Fettgewebes. Ein einfaches Maß für das Fettverteilungsmuster stellt dabei der Quotient von Taillen- und Hüftumfang dar. Danach ist es gesundheitlich bedenklich, wenn dieser Quotient bei Frauen über 0,85 und bei Männern über 1,00 liegt. Noch schneller lässt sich die abdominale Adipositas durch alleinige Messung des Bauchumfangs bestimmen. Das Krankheitsrisiko steigt bei einem Umfang von mehr als $80 \mathrm{~cm}$ bei Frauen und $94 \mathrm{~cm}$ bei Männern und ist deutlich erhöht bei Umfängen von mehr als 88 bzw. $102 \mathrm{~cm}$.

Die Ansammlung der Fettpolster in den Bauchorganen, speziell in der Leber, erhöht auch die Sterberaten ( $\triangleright$ Kap. 48). Ein spezielles Maß für die Einschätzung der Sterberisiken durch Übergewicht lautet ABSI ("a body shape index"). In seine Berechnung gehen zugleich Gewicht, Körpergröße und der Bauchumfang ein (Krakauer und Krakauer 2014).

\begin{tabular}{|c|c|c|}
\hline \multicolumn{3}{|c|}{$\begin{array}{l}- \text { Tab. } 43.1 \text { Stadieneinteilung des Körpergewichts } \\
\text { nach den Richtlinien der WHO }\end{array}$} \\
\hline \multicolumn{2}{|c|}{ Klassifikation } & Body-Mass-Index (BMI) \\
\hline \multicolumn{2}{|c|}{ Untergewicht } & $<18$ \\
\hline \multicolumn{2}{|c|}{ Normalgewicht } & $18,5-24,9$ \\
\hline \multicolumn{2}{|c|}{ Übergewicht } & $25-29,9$ \\
\hline \multirow[t]{3}{*}{ Adipositas } & - Grad I & $30-34,9$ \\
\hline & - Grad II & $35-40,0$ \\
\hline & - Grad III & $>40$ \\
\hline
\end{tabular}

$\mathrm{Zu}$ den vielen Schädigungsmöglichkeiten durch das viszerale Bauchfett ( $\triangleright$ Kap. 44) gehört nach den Ergebnissen von US-Studien mit Beobachtungszeiten von mehreren Jahrzehnten ebenfalls ein gesteigertes Risiko, an Demenz zu erkranken (Whitmer et al. 2008; Gelber et al. 2012). Besonders Übergewicht in den mittleren Lebensjahren erhöht dieses Risiko (Xu et al. 2011; Wotton und Godacre 2014). Nach Nebenauswertungen der Framingham Heart Study war das Hirnvolumen von ausgewählten Patienten umso niedriger, je höher ihr BMI lag (Debette et al. 2010).

Bei etwa einem Fünftel der Übergewichtigen bzw. Adipösen wachsen Zahl und Größe der Fettzellen genetisch bedingt gleichmäßig an und die Zellen verteilen sich breitflächig unter der gesamten Haut (Heid et al. 2010; Pulit et al. 2017). Es werden weniger der viszeralen Biomarker freigesetzt und damit fallen bei diesem Personenkreis die gewichtsbedingten Gesundheitseinschränkungen geringer aus. 